\title{
On Existence of Equilibria of Multi-port Linear AC Networks with Constant-Power Loads
}

\author{
Juan E. Machado, Robert Griñó, Senior Member, IEEE, Nikita Barabanov, Romeo Ortega, Fellow, IEEE, \\ and Boris Polyak,
}

\begin{abstract}
In this brief note we give an answer to the following question: Given a multi-port, linear AC network with instantaneous constant-power loads identify a set of active and reactive load powers for which there is no steady-state operating condition of the network-in this case we say that the power load is inadmissible. The identification is given in terms of feasibility of simple linear matrix inequalities, hence it can be easily verified with existing software. For one- or two-port networks the proposed feasibility test is necessary and sufficient for load power admissibility with the test for the former case depending only on the network data. Two benchmark numerical examples illustrate our results.
\end{abstract}

Index Terms-Constant power loads, AC LTI circuits, existence of equilibria.

\section{INTRODUCTION}

$\mathbf{T}$ HIS paper explores the problem of stability of linear time-invariant (LTI), multi-port AC circuits with instantaneous constant-power loads (CPLs), which model the behavior of some point-of-load converters. It is well-known that CPLs have a destabilizing effect that gives rise to significant oscillations or to network collapse, see [3], [4], [5], [7] and the references therein for a further discussion on the subject and [8] for a recent literature review. Clearly, a sine qua non condition for the correct behaviour of the network is the existence of a steady-state operating condition.

In this paper we give necessary conditions on the CPLs active and reactive powers for existence of equilibria for general multi-port networks. If these conditions are not satisfied the loads are called inadmissible. For one- or two-port

The work of Juan E. Machado was supported by the Government of México through Consejo Nacional de Ciencia y Tecnología (CONACyT). The work of R. Griñó was partially supported by the Government of Spain through the Ministerio de Economía y Competitividad Project DPI201341224-P and by the Generalitat de Catalunya through the project 2014SGR 267. R. Ortega, N. Barabanov and B. Polyak were supported by Government of Russian Federation (grant 074-U01, GOSZADANIE 2014/190 (project 2118)), the Ministry of Education and Science of Russian Federation (project 14.Z50.31.0031)

J.E. Machado and R. Ortega are with the Laboratory of Signals and Systems-CNRS/SUPELEC, 91190 Gif-Sur-Yvette, France (juan.machado[ortega]@12s.centralesupelec.fr).

R. Griñó is with Universitat Politecnica de Catalunya, Barcelona, Spain (roberto.grino@upc.edu).

N. Barabanov is with the Department of Mathematics, North Dakota State University, Fargo, ND 58105 USA, and with Department of Control Systems and Informatics, University ITMO, 49 Avenue Kronverskiy, 197101, Saint Petersburg, Russia (nikita.barabanov@ndsu.edu)

B. Polyak is with the Institute of Control Sciences and Energy Center, SkolTech, Moscow, Russia (boris@ipu.ru) networks with free reactive (or active) power these conditions are also sufficient-providing a full characterisation of the power that can be extracted from the AC network. The conditions are expressed in terms of feasibility of simple linear matrix inequalities (LMIs) for which reliable software is available. Moreover, for single-port networks the admissibility test depends only on the network data avoiding the need for an LMI analysis.

This work is an extension, to the case of AC networks, of our previous results for DC networks reported in [1], see also [7]. The extension is far from trivial because, on one hand, the mappings associated with the quadratic equations, whose solvability has to be studied in this problem, have complex domain and co-domain - in contrast with the ones of DC networks when these sets are real. Transferring these equations to the real domain results in twice the number of equations than unknowns stymying the application of the classical analytical tools used for DC networks, which treat the case of same number of equations and unknowns. On the other hand, the characterisation of the loads in AC networks involve its active and reactive component, whose simultaneous treatment complicates the nature of the mathematical problem to be solved.

The remainder of the paper is structured as follows. In Section II the problem addressed in the paper is formulated and the difference with the DC networks case is highlighted. Section III contains three lemmata instrumental for the establishment of the results. Section IV gives necessary conditions for existence of equilibria for multi-port networks. In Section $\mathrm{V}$ we give necessary and sufficient conditions for the case of one- or two-port networks. In Section VI we apply the previous results to provide a characterization of the admissible and inadmissible loads, while in Section VII we illustrate the results with two benchmark examples. We wrap-up the paper with concluding remarks and future research in Section VIII. To enhance readability all proofs are given in appendices at the end of the paper.

\section{Problem Formulation}

\section{A. Mathematical model of AC networks with CPLs}

In this note we deal with LTI AC electrical networks with CPLs working in sinusoidal steady-state at a frequency $\omega_{0} \in$ $\mathbb{R}_{+}$, see Fig. 1. The description of this regime in the frequency domain is

$$
V\left(j \omega_{0}\right)=G\left(j \omega_{0}\right) I\left(j \omega_{0}\right)+K\left(j \omega_{0}\right),
$$


where $^{1} V \in \mathbb{C}^{m}$ and $I \in \mathbb{C}^{m}$ are the vectors of generalized Fourier transforms of the port voltages and currents, respectively, and $K \in \mathbb{C}^{m}$ captures the effect of the external (current or voltage) AC sources, all evaluated at the frequency $\omega_{0}$. Equation (1) can also be seen as the Thevenin equivalent model of the $m$-port AC linear network including the voltage and current sources and, then, $G \in \mathbb{C}^{m \times m}$ should be interpreted as the frequency domain impedance matrix of the network at the frequency $\omega_{0}$.

It should be underscored that, since $G(s) \in \mathbb{R}^{m \times m}(s)$ is the impedance of an LTI circuit it is strictly positive real [in the circuit theory sense] [2], consequently it satisfies

$$
G(j \omega)+G^{\mathrm{H}}(j \omega)>0, \forall \omega \in \mathbb{R},
$$

where $(\cdot)^{\mathrm{H}}$ denotes the complex conjugate transpose.

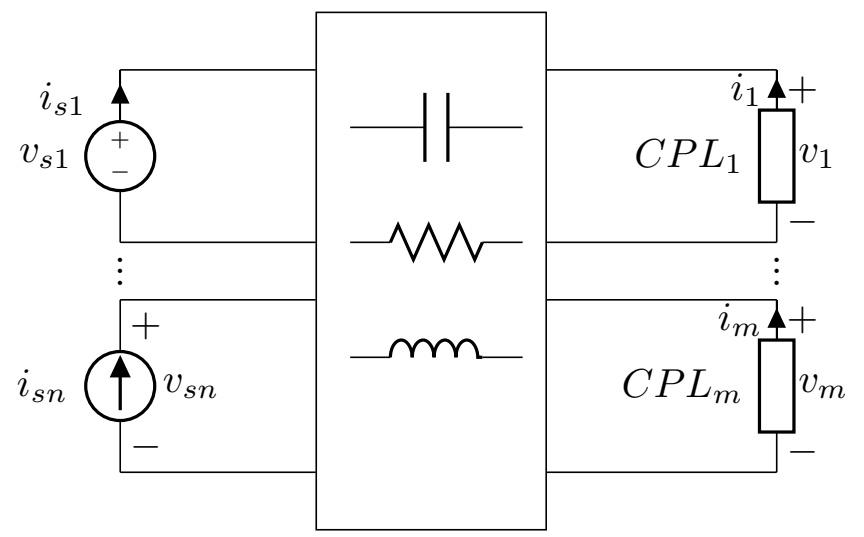

Fig. 1. Schematic representation of multi-port AC LTI electrical networks with $n$ external voltage and current sources feeding $m$ CPLs.

Defining the apparent power at the CPLs $S_{i} \in \mathbb{C}$ as

$$
S_{i}:=P_{i}+j Q_{i}, \quad i \in\{1, \ldots m\},
$$

where $P_{i} \in \mathbb{R}$ and $Q_{i} \in \mathbb{R}$ are the active and reactive power at port $i$, respectively, the CPLs constraint the network via

$$
V_{i} I_{i}^{*}=-S_{i},
$$

where $(\cdot)^{*}$ is the complex conjugate. In (4), and throughout the rest of the paper, the qualifier $i \in\{1, \ldots m\}$ is omitted.

Obviously, a necessary and sufficient condition for the existence of a sinusoidal steady-state (at a given frequency $\omega_{0}$ ) is that the complex equations (1), (3) and (4) have a solution, which is the question we address in this paper.

\section{B. Compact representation and comparison with DC networks}

We make the important observation that, eliminating the voltage vector $V$, the system of equations (1), (3) and (4) can be compactly represented by the set of complex quadratic equations

$$
f_{i}(I)=0
$$

\footnotetext{
${ }^{1}$ To simplify the notation the argument $j \omega_{0}$ is omitted in the sequel.
}

where the complex mappings $f_{i}: \mathbb{C}^{m} \rightarrow \mathbb{C}$ are defined as

$$
f_{i}(I):=I^{\mathrm{H}}\left(e_{i} e_{i}^{\top} G\right) I+I^{\mathrm{H}} K_{i} e_{i}+S_{i},
$$

where $e_{i} \in \mathbb{R}^{m}$ is the $i$-th Euclidean basis vector. The fact that the mappings $f_{i}(\cdot)$ have complex domain and co-domain represents a major technical difficulty to establish conditions for existence of solutions of (5), (6).

This situation should be contrasted with the case of DC networks where the mappings have real domain and codomain. Indeed, as shown in [1], there exists a constant steadystate if and only if the real quadratic equations $\vartheta_{i}(\mathrm{I})=0$ have a real solution, with the mappings $\vartheta_{i}: \mathbb{R}^{m} \rightarrow \mathbb{R}$ given by

$$
\vartheta_{i}(\mathrm{I}):=\mathrm{I}^{\top}\left[e_{i} e_{i}^{\top} G(0)\right] \mathrm{I}+\mathrm{I}^{\top} \mathbf{s}_{i} e_{i}+P_{i},
$$

where $\mathrm{I} \in \mathbb{R}^{m}$ are the currents, $G(0) \in \mathbb{R}^{m \times m}$ is the DC gain of the impedance matrix, and $\mathrm{s}_{i} \in \mathbb{R}$ are the external (voltage or current) DC sources.

\section{Considered scenario and characterisation of the loads}

Finding conditions for solvability of the equations (5), (6), for arbitrary $P_{i}, Q_{i}, G$ and $K$, is a nonlinear analysis daunting task, which is also of little practical interest. Indeed, in practical scenarios $P_{i}$ and-or $Q_{i}$ are fixed and we want to find conditions on $G$ and $K$ such that an equilibrium existsinterestingly, we show below that this makes the problem mathematically tractable. The powers $P_{i}$ and $Q_{i}$ for which an equilibrium exists are said to be admissible, otherwise, they are called inadmissible.

To state in a compact manner the problem formulation we define the vectors

$$
\begin{aligned}
P & :=\operatorname{col}\left(P_{1}, \ldots, P_{m}\right) \in \mathbb{R}^{m} \\
Q & :=\operatorname{col}\left(Q_{1}, \ldots, Q_{m}\right) \in \mathbb{R}^{m} \\
S & :=\operatorname{col}\left(S_{1}, \ldots, S_{m}\right) \in \mathbb{C}^{m},
\end{aligned}
$$

which are assumed given, and we want to find conditions on $G$ and $K$ that imply that $P$ and-or $Q$ belong to either one of the following sets.

- $\mathcal{P}_{\mathrm{FAQ}}^{\mathrm{I}} \subset \mathbb{R}^{m}$ set of $P$ that are inadmissible for all $Q$. That is, if $P \in \mathcal{P}_{\mathrm{FAQ}}^{\mathrm{I}}$ there is no steady-state no matter what $Q$ is.

- $\mathcal{Q}_{\mathrm{FAP}}^{\mathrm{I}} \subset \mathbb{R}^{m}$ set of $Q$ that are inadmissible for all $P$. That is, if $Q \in \mathcal{Q}_{\mathrm{FAP}}^{\mathrm{I}}$ there is no steady-state no matter what $P$ is.

- $\mathcal{S}^{\mathrm{I}} \subset \mathbb{R}^{m} \times \mathbb{R}^{m}$ set of $(P, Q)$ that are inadmissible. That is, if $(P, Q) \in \mathcal{S}^{\mathrm{I}}$ there is no steady-state.

Our first contribution is the definition of LMIsparameterised in $P$ and $Q$-whose feasibility implies that $P$ and-or $Q$ belong to either one of the aforementioned sets.

A second contribution is that, for the case of one- or twoport networks, i.e., $m \leq 2$, we provide a full characterization of the following sets.

- $\mathcal{P}_{\mathrm{FSQ}}^{\mathrm{A}} \subset \mathbb{R}^{m}$ set of $P$ that are admissible for some $Q$. That is, if $P \in \mathcal{P}_{\mathrm{FSQ}}^{\mathrm{A}}$ there is a $Q$ (that can be computed) for which there is a steady-state.

- $\mathcal{Q}_{\mathrm{FSP}}^{\mathrm{A}} \subset \mathbb{R}^{m}$ set of $Q$ that are admissible for some $P$. That is, if $Q \in \mathcal{P}_{\mathrm{FSP}}^{\mathrm{A}}$ there is a $P$ (that can be computed) for which there is a steady-state. 
- (For $m=1) \mathcal{S}^{\mathrm{A}} \subset \mathbb{R}^{m} \times \mathbb{R}^{m}$ set of $(P, Q)$ that are admissible. That is, if $(P, Q) \in \mathcal{S}^{\mathrm{A}}$ there is a steady-state.

By full characterisation of the sets we mean that

$$
\begin{aligned}
& \mathcal{P}_{\mathrm{FSQ}}^{\mathrm{A}} \cup \mathcal{P}_{\mathrm{FAQ}}^{\mathrm{I}}=\mathbb{R}^{m} \quad(\text { for } m \leq 2) \\
& \mathcal{Q}_{\mathrm{FSP}}^{\mathrm{A}} \cup \mathcal{Q}_{\mathrm{FAP}}^{\mathrm{I}}=\mathbb{R}^{m} \quad(\text { for } m \leq 2) \\
& \mathcal{S}^{\mathrm{A}} \cup \mathcal{S}^{\mathrm{I}}=\mathbb{R}^{m} \times \mathbb{R}^{m} \quad(\text { for } m=1 \text { ). }
\end{aligned}
$$

In other words, that the conditions of admissibility for $P$ or $Q$ are necessary and sufficient.

From the practical viewpoint the inadmissibility sets $\mathcal{P}_{\text {FAQ }}^{I}$ and $\mathcal{Q}_{\mathrm{FAP}}^{\mathrm{I}}$ allows us to rule out "bad" $P s$ and $Q s$, respectively. The set $\mathcal{S}^{\mathrm{I}}$ is useful in the scenario when the devices at the ports transfer constant power with a specified power factor $\mathrm{PF}_{\mathrm{i}}=\frac{P_{i}}{S_{i}}$, in which case we fix $S$. Another scenario of practical interest where the set $\mathcal{S}^{\mathrm{I}}$ is instrumental is when $P$ is fixed (possibly some elements zero) and some $Q_{i}$ are fixed and the others are free. The main question in this case is if some specific values of the free $Q_{i}$ can enlarge the set of admissible $P$.

Finally, for $m \leq 2$, the sets $\mathcal{P}_{\mathrm{FSQ}}^{\mathrm{A}}$ and $\mathcal{Q}_{\mathrm{FSP}}^{\mathrm{A}}$ provide a complete answer to the admissibility question when $P$ is fixed and $Q$ is free and, vice versa, when $Q$ is fixed and $P$ is free, respectively. Additionally, for the special case $m=1$ we provide a full characterization of the set $\mathcal{S}^{\mathrm{A}}$. See Section $\mathrm{V}$ for an illustration of these scenarios in two numerical examples.

\section{ThreE PRELIMINARY LEMMATA}

In this section we present three lemmata that are instrumental to establish our results. The first lemma shows that the $m$ complex quadratic equations (5), (6) admit a solution if and only if a system of $2 m$ real quadratic equations with $2 m$ unknowns admit a solution. Due to the fact that in AC networks the complex power $S$ has active $P$ and reactive $Q$ components gives rise to a new situation where the number of equations is different from the number of unknowns. The second lemma gives necessary conditions for the solvability of such a system of equations and is an extension of Lemma 1 of [1] when these numbers are the same. Finally, the third lemma shows that these conditions are also sufficient if the number of equations is smaller than three-provided an additional assumption is verified. The latter is always holds true in DC networks, but it has to be verified in the AC case.

\section{A. A real representation of (5), (6)}

To streamline the presentation of the following lemma we define the real mappings $g_{i}, h_{i}: \mathbb{R}^{2 m} \rightarrow \mathbb{R}$

$$
\begin{aligned}
& g_{i}(d):=d^{\top} \hat{A}_{i} d+d^{\top} \hat{b}_{i}+2 P_{i} \\
& h_{i}(d):=d^{\top} \hat{B}_{i} d+d^{\top} \hat{q}_{i}+2 Q_{i},
\end{aligned}
$$

where

$$
\begin{aligned}
d & :=\left[\begin{array}{c}
\operatorname{Re}\{I\} \\
\operatorname{Im}\{I\}
\end{array}\right], \hat{b}_{i}:=\left[\begin{array}{c}
\operatorname{Re}\{K\} e_{i} \\
\operatorname{Im}\{K\} e_{i}
\end{array}\right], \hat{q}_{i}:=\left[\begin{array}{c}
\operatorname{Im}\{K\} e_{i} \\
-\operatorname{Re}\{K\} e_{i}
\end{array}\right] \\
\hat{A}_{i}: & =\left[\begin{array}{cc}
e_{i} e_{i}^{\top}\left(\operatorname{Re}\{G\}+\operatorname{Re}\{G\}^{\top}\right) & e_{i} e_{i}^{\top}\left(-\operatorname{Im}\{G\}+\operatorname{Im}\{G\}^{\top}\right) \\
e_{i} e_{i}^{\top}\left(\operatorname{Im}\{G\}-\operatorname{Im}\{G\}^{\top}\right) & e_{i} e_{i}^{\top}\left(\operatorname{Re}\{G\}+\operatorname{Re}\{G\}^{\top}\right)
\end{array}\right] \\
\hat{B}_{i} & :=\left[\begin{array}{cc}
e_{i} e_{i}^{\top}\left(\operatorname{Im}\{G\}+\operatorname{Im}\{G\}^{\top}\right) & e_{i} e_{i}^{\top}\left(\operatorname{Re}\{G\}-\operatorname{Re}\{G\}^{\top}\right) \\
e_{i} e_{i}^{\top}\left(-\operatorname{Re}\{G\}+\operatorname{Re}\{G\}^{\top}\right) & e_{i} e_{i}^{\top}\left(\operatorname{Im}\{G\}+\operatorname{Im}\{G\}^{\top}\right)
\end{array}\right] .
\end{aligned}
$$

Lemma 1: The set of complex mappings $f_{i}: \mathbb{C}^{m} \rightarrow \mathbb{C}$ given in (6) verifies

$$
\begin{aligned}
g_{i}(d) & =\operatorname{Re}\left\{f_{i}(I)\right\} \\
h_{i}(d) & =\operatorname{Im}\left\{f_{i}(I)\right\} .
\end{aligned}
$$

Consequently,

$$
\exists I \in \mathbb{C}^{m}\left|f_{i}(I)=0 \Leftrightarrow \exists d \in \mathbb{R}^{2 m}\right| g_{i}(d)=h_{i}(d)=0 .
$$

The proof of this lemma is established by direct, but lengthy, computations and is omitted for brevity.

\section{B. Necessary condition for the solution of quadratic equations}

Lemma 2: Consider the real mappings $v_{k}: \mathbb{R}^{n} \rightarrow \mathbb{R}$ where ${ }^{2}$ $k \in \bar{\ell}:=\{1,2, \ldots, \ell\}$,

$$
v_{k}(x):=x^{\top} A_{k} x+2 x^{\top} b_{k}+c_{k},
$$

$x \in \mathbb{R}^{n}, b_{k} \in \mathbb{R}^{n}, c_{k} \in \mathbb{R}$, and $A_{k}$ are symmetric $n \times n$ matrices with $n \geq 2$ [non-necessarily equal to $\ell$ ]. Define the following $(n+1) \times(n+1)$ real matrices

$$
\mathcal{A}_{k}:=\left[\begin{array}{ll}
A_{k} & b_{k} \\
b_{k}^{\top} & c_{k}
\end{array}\right] .
$$

The following implication is true:

$$
\exists t_{k} \in \mathbb{R} \mid \sum_{k=1}^{\ell} t_{k} \mathcal{A}_{k}>0 \Rightarrow\left\{x \in \mathbb{R}^{n} \mid v_{k}(x)=0\right\}=\varnothing \text {. }
$$

Remark 1: Lemma 2, which gives necessary conditions for existence of solutions of $\ell$ quadratic equations with $n$ unknowns, is an extension of Lemma 1 of [1] where the particular case $n=\ell$ is treated.

\section{Sufficient condition for the solution of two equations}

Lemma 3: Consider two mappings $v_{1}(x), v_{2}(x)$ as given in (11) and two matrices $\mathcal{A}_{1}, \mathcal{A}_{2}$ as in (12). Assume there exists $s_{1}, s_{2} \in \mathbb{R}$, such that

$$
s_{1} A_{1}+s_{2} A_{2}>0 .
$$

The following implication is true:

$$
\begin{array}{r}
\exists t_{1}, t_{2} \in \mathbb{R} \mid t_{1} \mathcal{A}_{1}+t_{2} \mathcal{A}_{2}>0 \\
\Leftarrow\left\{x \in \mathbb{R}^{n} \mid v_{1}(x)=v_{2}(x)=0\right\}=\varnothing .
\end{array}
$$

Remark 2: Lemma 3, which gives sufficient conditions for existence of solutions of two quadratic equations with $n$ unknowns, is related with Proposition 3 of [1] where the condition (14) is not explicitly stated because it is always satisfied in DC networks. However, as explained in Section V, this is not always the case for AC networks. Notice that the need for (14) is clearly stated in [6, Theorem 2.2]. Moreover, as indicated in Remark 1, in [1, Proposition 3] only the particular case $n=\ell$ is treated.

Remark 3: Invoking Lemma 2 we see that the "only if" condition (15) for two-port equations is actually and "if and only if"-with the "if" part holding even without (14).

\footnotetext{
${ }^{2}$ In the sequel we omit the clarification that $k \in \bar{l}$.
} 


\section{Necessary CONDitions fOR EXISTENCE OF A STEADY-STATE FOR $m$-PORT NETWORKS}

A direct application of Lemmata 1 and 2 provides a way to determine the inadmissibility of $P$ and-or $Q$ from the feasibility of parameterised LMIs. Also, we give an interpretation of the results in terms of the extracted active power.

\section{A. An LMI-based inadmissibility condition}

Proposition 1: Fix $P, Q \in \mathbb{R}^{m}$. If there exist $T=$ $\operatorname{diag}\left\{t_{i}\right\}_{i=1}^{m} \in \mathbb{R}^{m \times m}$ and $\bar{T}=\operatorname{diag}\left\{\bar{t}_{i}\right\}_{i=1}^{m} \in \mathbb{R}^{m \times m}$ such that

$$
R_{P}(T)+R_{Q}(\bar{T})>0,
$$

where $R_{P}(T)$ and $R_{Q}(\bar{T})$ are $(2 m+1) \times(2 m+1)$ real matrices given by

$R_{P}(T):=\left[\begin{array}{ccc}T \operatorname{Re}\{G\}+\operatorname{Re}\{G\}^{\top} T & -T \operatorname{Im}\{G\}+\operatorname{Im}\{G\}^{\top} T & T \operatorname{Re}\{K\} \\ T \operatorname{Im}\{G\}-\operatorname{Im}\{G\}^{\top} T & T \operatorname{Re}\{G\}+\operatorname{Re}\{G\}^{\top} T & T \operatorname{Im}\{K\} \\ \operatorname{Re}\{K\}^{\top} T & \operatorname{Im}\{K\}^{\top} T & 2 P^{\top} T \mathbf{1}_{m}\end{array}\right]$

and

$R_{Q}(\bar{T}):=\left[\begin{array}{cc}\bar{T} \operatorname{Im}\{G\}+\operatorname{Im}\{G\}^{\top} \bar{T} & \bar{T} \operatorname{Re}\{G\}-\operatorname{Re}\{G\}^{\top} \bar{T} \\ -\bar{T} \operatorname{Re}\{G\}+\operatorname{Re}\{G\}^{\top} \bar{T} & \bar{T} \operatorname{Im}\{G\}+\operatorname{Im}\{G\}^{\top} \bar{T} \\ \operatorname{Im}\{K\}^{\top} \bar{T} & -\operatorname{Re}\{K\}^{\top} \bar{T}\end{array}\right.$

$\bar{T} \operatorname{Im}\{K\}$ $-\bar{T} \operatorname{Re}\{K\}$ $2 Q^{\top} \bar{T} \mathbf{1}_{m}$

Then, there is no sinusoidal steady-state for the system.

Remark 4: In Proposition 1 the values of $P$ and $Q$ are fixed a priori, then the positivity condition (16) is a simple LMI in $(T, \bar{T})$ for which reliable software is available. Otherwise, it represents a bilinear matrix inequality in $(T, \bar{T}, P, Q)$, whose solution is far from trivial.

Remark 5: Defining ${ }^{3} \tilde{P}:=P^{\top} T$ and $\tilde{Q}:=Q^{\top} \bar{T}$ it is possible to reparameterize the matrices $R_{P}$ and $R_{Q}$ to transform (16) in an LMI in $(T, \tilde{P}, \bar{T}, \tilde{Q})$. However, as discussed at the beginning of Subsection II-C, this formulation is of little practical interest.

\section{B. Bound on the extracted active power}

In this subsection we assume that the active power flows only from the network to the loads and give an upper bound on the admissible overall extracted power.

To streamline the result we define the $2 m \times 2 m$ real, symmetric matrix

$$
M:=\left[\begin{array}{cc}
\operatorname{Re}\{G\}+\operatorname{Re}\{G\}^{\top} & -\operatorname{Im}\{G\}+\operatorname{Im}\{G\}^{\top} \\
-\operatorname{Im}\{G\}^{\top}+\operatorname{Im}\{G\} & \operatorname{Re}\{G\}+\operatorname{Re}\{G\}^{\top}
\end{array}\right],
$$

that, in view of (2), is positive definite.

Proposition 2: Suppose that all CPLs extract active power from the network, that is $P_{i} \geq 0$. A necessary condition for the existence of a sinusoidal steady-state is that the overall extracted power is upper bounded as follows

$$
\sum_{i=1}^{m} P_{i} \leq \frac{1}{2}\left[\begin{array}{l}
\operatorname{Re}\{K\} \\
\operatorname{Im}\{K\}
\end{array}\right]^{\top} M^{-1}\left[\begin{array}{l}
\operatorname{Re}\{K\} \\
\operatorname{Im}\{K\}
\end{array}\right]
$$

\footnotetext{
${ }^{3}$ We thank the anonymous reviewer for this remark.
}

The condition above is similar to the necessary condition for existence of a constant steady-state regime for LTI DC networks with CPLs presented in [1, Proposition 2]. The condition for DC networks is the existence of a positive definite diagonal matrix $T$ such that

$$
\sum_{i=1}^{m} t_{i} P_{i} \leq \frac{1}{2}(T K)^{\top}\left[T G(0)+G^{\top}(0) T\right]^{-1} T K .
$$

To compare this bound with (20) recall that in the DC case $\omega_{0}=0$ and the vector of external sources $K$ is real-whose elements were denoted as $s_{i}$ in (7). Therefore, (20) reduces to (21) but with $T=I_{m}$. The presence of the free matrix $T$ makes the bound for DC networks tighter.

\section{NeCESSARY AND SUFFICIENT LOAD AdMISSIBILITY CONDITIONS FOR ONE- OR TWO-PORT NETWORKS}

To make the conditions for existence of a steady-state not only necessary but also sufficient we consider in this section the case of one- or two-port networks, i.e., $m \leq 2$. In Proposition 3 of [1] a similar scenario is treated for DC networks. However, as indicated in Remark 2, this proposition is inapplicable in the $\mathrm{AC}$ case and we need to invoke Lemma 3 that, we recall, requires the verification of condition (14).

As done in the previous section, we consider the scenarios where $P$ is fixed and let $Q$ free or, vice versa, where $Q$ is fixed and $P$ is free and, lastly, for the particular case of $m=1$ we take both $P$ and $Q$ fixed. We present first the latter case since it is a natural complement to Proposition 1 [for $m=1$ ].

\section{A. Single-port networks with fixed active and reactive power}

Our next result pertains to single-port networks and gives two different necessary and sufficient conditions for a pair $(P, Q)$ to be admissible. The first condition is given in the spirit of Proposition 1, that is, it relates the existence of a sinusoidal steady-state with the feasibility of an LMI. In addition, a radically different new condition, is given exclusively in terms of the data of the problem.

Proposition 3: For a one-port network fix $P$ and $Q$. The following three statements are equivalent.

- The system admits a sinusoidal steady-state.

- There are no real scalars $T$ and $\bar{T}$ such that

$$
R_{P}(T)+R_{Q}(\bar{T})>0 .
$$

- The inequality

$$
|K|^{2} \geq 2\left(|S||G|+\operatorname{Re}\left\{S^{*} G\right\}\right),
$$

holds true, where $|\cdot|$ is the magnitude of the complex number.

It is important to underscore that inequality (23) is written only in terms of the original parameters of the system, that is $G, K$ and $S$, and it does not include additional variables. Also, this inequality reduces to the necessary and sufficient condition for existence of equilibria for LTI DC circuits for the case $m=1$ presented in [7, Section II]. 


\section{B. Two-port networks with free active or reactive power}

Suppose that the network is constrained to satisfy the active power demand but the reactive power is unconstrained, i.e., the reactive power term $Q$ can be arbitrarily assigned. Now, as seen from (8), the quadratic mappings $g_{i}(d)$ are independent of the reactive power $Q$ and the quadratic mappings $h_{i}(d)$ are independent of the active power $P$. Since the $Q$ is free the equations $h_{i}(d)=0$ are trivialised, reducing to the definition

$$
Q_{i}:=-\frac{1}{2}\left(\bar{d}^{\top} \hat{B}_{i} \bar{d}+\bar{d}^{\top} \hat{c}_{i}\right),
$$

where $\bar{d} \in \mathbb{R}^{2 m}$ is the solution of the equations $g_{i}(\bar{d})=0$. The case where the network is constrained to satisfy a given reactive power, with unconstrained active power, is analogous to the scenario just described and now the existence of a sinusoidal steady-state is equivalent to the solvability of the system $h_{i}(d)=0$.

Now, for two-port networks there are only two quadratic equations, in which case, Lemma 3 states that their solvability is equivalent to the feasibility of its associated LMI—provided condition (14) is satisfied.

Proposition 4: For a two-port network, fix $P$ and suppose $Q$ is unconstrained. The following two statements are equivalent.

- The network admits a sinusoidal steady-state.

- There is no diagonal matrix $T$ such that $R_{P}(T)>0$.

Proposition 5: For a two-port network, fix $Q$ and suppose $P$ unconstrained. Assume there exists $\hat{s}_{1}, \hat{s}_{2} \in \mathbb{R}$, such that

$$
\hat{s}_{1} \hat{B}_{1}+\hat{s}_{2} \hat{B}_{2}>0 \text {. }
$$

The following two statements are equivalent.

- The network admits a sinusoidal steady-state.

- There is no diagonal matrix $\bar{T}$ such that $R_{Q}(\bar{T})>0$.

Remark 6: Notice from the propositions above that for the case of fixed reactive power the additional assumption (24) is imposed on the network. This assumption is absent when we fix the active power-a distinction that is clarified in the proofs. It is worth remarking that (24) is verified in both benchmark examples given in Section VI.

Remark 7: Unfortunately, for $m \geq 2$ we do not provide necessary and sufficient conditions for power load admissibility when both $P$ and $Q$ are fixed. This stems from the fact that the proof of sufficiency relies, either on Finsler's Lemma as in Lemma 3 or on establishing convexity of the image of the mapping defined by the quadratic equations as done in [1, Proposition 3]. To the extent of our knowledge, there are no general results concerning the (global) convexity of these mappings nor extensions of Finsler's Lemma when there are more than two equations. As explained in Subsection II-B this corresponds in the AC case to single-port networks, while in the DC case is applicable to two-port networks.

Remark 8: A necessary and sufficient condition for power load admissibility for two-port networks can be established under some particular conditions. For instance, if one of the elements of the vector $K$ is zero and the corresponding element of $Q$ is zero, this condition is the feasibility of the parameterised LMI

$$
\exists \tau_{1}, \tau_{2} \in \mathbb{R} \mid \tau_{1} F_{1}(p)+\tau_{2} F_{2}(p)>0, \forall p \in \mathbb{R}^{3},
$$

where $F_{1}, F_{2}: \mathbb{R}^{3} \rightarrow \mathbb{R}$ are quadratic equations of the form

$$
F_{j}(p):=p^{\top} \alpha_{j} p+p^{\top} \beta_{j}+\gamma_{j}, j=1,2,
$$

where $\alpha_{j} \in \mathbb{R}^{2 \times 2}, \beta_{j} \in \mathbb{R}^{2}$ and $\gamma_{j} \in \mathbb{R}$ are computable from $(G, K, P, Q)$. The precise statements and proofs are extremely involved and may be found in an extended version of this paper, which is available upon request to the authors.

\section{IDENTIFICATION OF THE ADMISSIBILITY AND INADMISSIBILITY SETS FOR THE LOADS}

In this section we apply the results of Propositions 1 and 3-5 to identify the sets that characterise the admissible and inadmissible loads described in Subsection II-C.

\section{A. Inadmissibility sets for m-port networks}

The identification of the inadmissibility sets $\mathcal{P}_{\text {FAQ }}^{I}, \mathcal{Q}_{\mathrm{FAP}}^{\mathrm{I}}$ and $\mathcal{S}^{\mathrm{I}}$ follows as a direct corollary of Proposition 1. First, notice that $R_{P}(0)=0$ and $R_{Q}(0)=0$. Therefore, setting $\bar{T}=0$ in (16), $R_{P}(T)>0$ implies the non-existence of a sinusoidal steady-state for the system - with a similar situation for $R_{Q}(\bar{T})>0$ and $T=0$. Second, matrix $R_{P}$ is independent of $Q$ and matrix $R_{Q}$ is independent of $P$. On the other hand, matrix $R_{P}(T)+R_{Q}(\bar{T})$ is dependent on both $P$ and $Q$ simultaneously.

In view of the observations above it is clear that the following implications hold:

- $\exists T$ such that $R_{P}(T)>0 \Rightarrow P \in \mathcal{P}_{\text {FAQ }}^{\mathrm{I}}$

- $\exists \bar{T}$ such that $R_{Q}(\bar{T})>0 \Rightarrow Q \in \mathcal{Q}_{\mathrm{FAP}}^{\mathrm{I}}$

- $\exists T, \bar{T}$ such that $R_{P}(T)+R_{Q}(\bar{T})>0 \Rightarrow(P, Q) \in \mathcal{S}^{\mathrm{I}}$.

\section{B. Admissibility sets for one- or two-port networks}

The following characterization for the admissibility sets described in Section II follows directly from Propositions 3-5.

- $(m \leq 2) \nexists T$ such that $R_{P}(T)>0 \Leftrightarrow P \in \mathcal{P}_{\mathrm{FSQ}}^{\mathrm{A}}$.

- $(m \leq 2) \nexists \bar{T}$ such that $R_{Q}(\bar{T})>0 \Leftrightarrow Q \in \mathcal{Q}_{\mathrm{FSP}}^{\mathrm{A}}$.

- $(m=1) \nexists T, \bar{T}$ such that $R_{P}(T)+R_{Q}(\bar{T})>0 \Leftrightarrow$ $(P, Q) \in \mathcal{S}^{\mathrm{A}}$.

- $(m=1)(23)$ holds $\Leftrightarrow(P, Q) \in \mathcal{S}^{\mathrm{A}}$.

\section{Two Illustrative Examples}

\section{A. A single-port RLC circuit}

The linear RLC circuit shown in Fig. 2 has been previously used in studies with CPLs in [1] but considering a constant voltage source instead of a sinusoidal AC voltage source as in the present work. If we define the voltage source as

$$
v_{g}=\sqrt{2} V_{g} \cos \left(\omega_{0} t\right) \mathrm{V},
$$

then $G$ and $K$, evaluated a $j \omega_{0}$, are given by

$$
\begin{aligned}
& G=\frac{r_{1}+j L_{1} w_{0}}{\left(1+\frac{r_{1}}{r_{c}}-L_{1} C_{1} w_{0}^{2}\right)+j\left(r_{1} C_{1}+\frac{L_{1}}{r_{c}}\right) w_{0}} \\
& K=\frac{V_{g}}{\left(1+\frac{r_{1}}{r_{c}}-L_{1} C_{1} w_{0}^{2}\right)+j\left(r_{1} C_{1}+\frac{L_{1}}{r_{c}}\right) w_{0}} .
\end{aligned}
$$


Using the circuit parameters from Table I, and with the particular value of $r_{c}=5 \mathrm{k} \Omega$, the above expressions result in

$$
G=0.0412+j 0.0238, \quad K=24.3592-j 0.6219 .
$$

Since $m=1$, Proposition 3 gives a full characterization of the set $\mathcal{S}^{\mathrm{A}}$ for this circuit either in terms of the feasibility of the LMI (22) or via the simple inequality (23).

Fig. 3 shows the feasibility and infeasibility regions on the $P-Q$ plane for the condition given in (22). The graph was obtained by taking fixed values of $P$ and $Q$ in the discretized set $[0,2500] \times[-6000,5000]$. According to Proposition 3, any pair $(P, Q)$ in the infeasibility region corresponds to an admissible pair, i.e., $(P, Q) \in \mathcal{S}^{\mathrm{A}}$. On the other hand, any pair $(P, Q)$ in the feasibility region corresponds to an inadmissible pair, i.e., $(P, Q) \in \mathcal{S}^{\mathrm{I}}$.

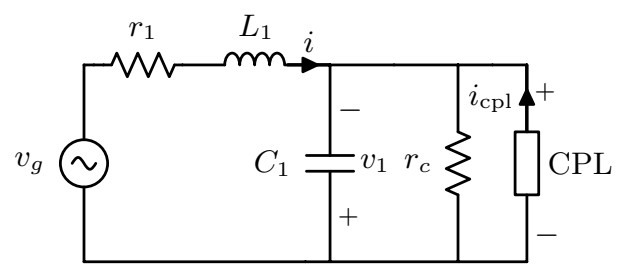

Fig. 2. AC Linear RLC circuit with a CPL.

TABLE I

PARAMETERS FOR THE CIRCUIT IN FIGS. 2 AND 5

\begin{tabular}{c|c|c|c}
\hline \hline$r_{1}=0.04 \Omega$ & $L_{1}=78.0 \mu \mathrm{H}$ & $C_{1}=2.0 \mathrm{mF}$ & $V_{g}=24 \mathrm{~V}$ \\
\hline$r_{2}=0.06 \Omega$ & $L_{2}=98.0 \mu \mathrm{H}$ & $C_{2}=1.0 \mathrm{mF}$ & $\omega_{0}=2 \pi 50 \mathrm{rad} / \mathrm{s}$ \\
\hline \hline
\end{tabular}

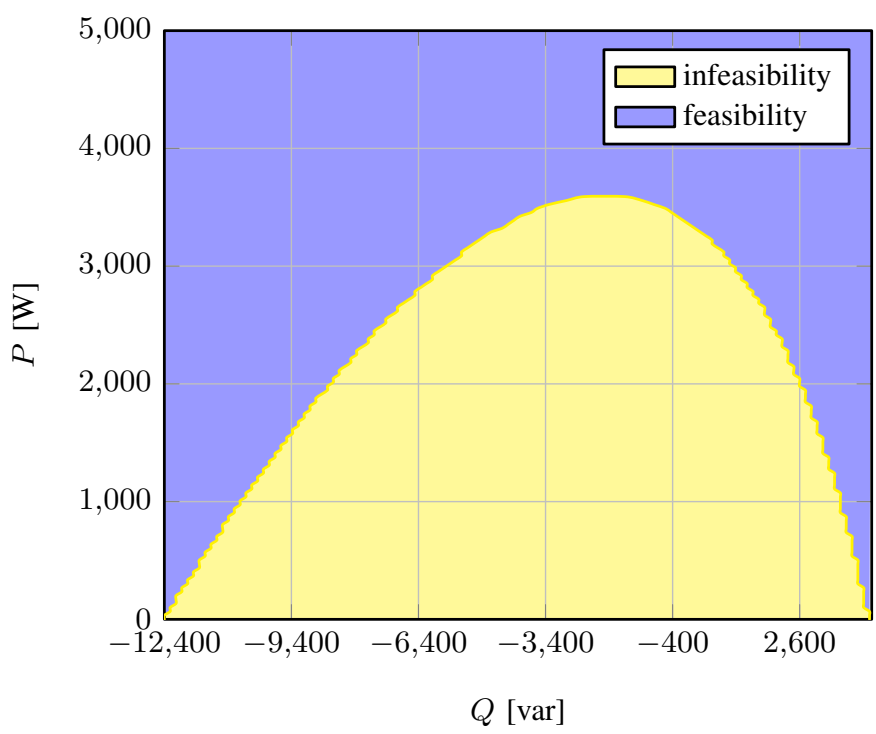

Fig. 3. Feasibility and infeasibility regions of $R_{P}(T)+$ $R_{Q}(\bar{T})>0$ for the circuit of Fig. 2 .
In Fig. 4 we plot the exact boundary of existence of solutions of $f_{i}(I)=0$ - that was computed numerically-and the feasibility boundary of the inequality (23); as predicted by the theory they are identical and also coincide with the boundary of Fig. 3 .

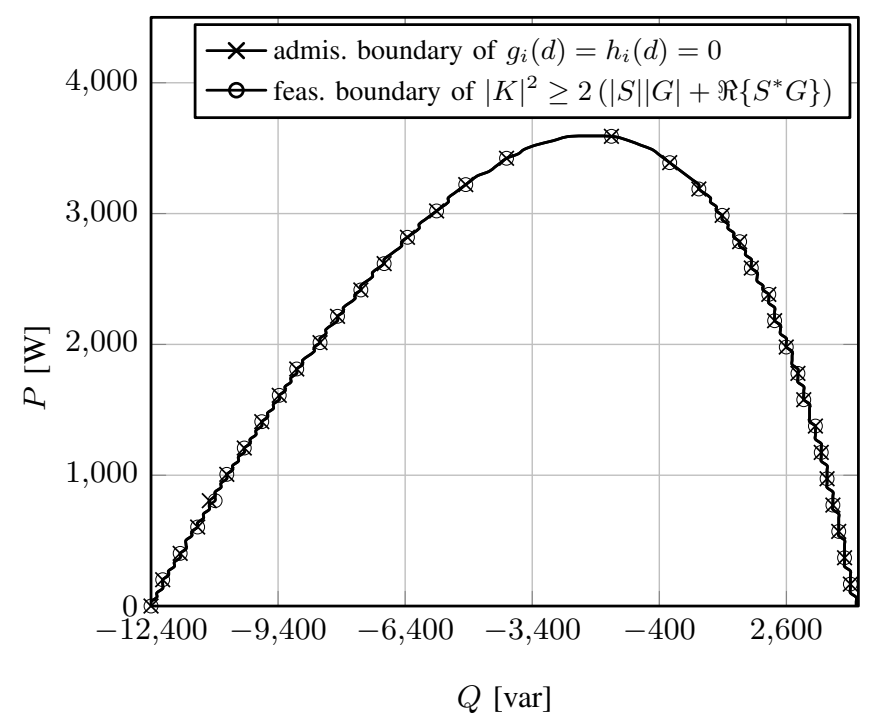

Fig. 4. Boundary of the admissibility set $\mathcal{S}^{\mathrm{A}}$ and feasibility boundary of the inequality $|K|^{2} \geq 2\left(|S||G|+\Re\left\{S^{*} G\right\}\right)$.

\section{B. A two-port system}

Fig. 5 shows an LTI circuit with two CPLs and the AC source (25). In this case, the matrix $G$ and the vector $K$ are

$$
\begin{aligned}
& G=\frac{1}{d\left(j \omega_{0}\right)}\left[\begin{array}{ll}
n_{11}\left(j \omega_{0}\right) & n_{12}\left(j \omega_{0}\right) \\
n_{21}\left(j \omega_{0}\right) & n_{22}\left(j \omega_{0}\right)
\end{array}\right], \\
& K=\frac{V_{g}}{d\left(j \omega_{0}\right)}\left[\begin{array}{c}
\left(\left(1-\omega_{0}^{2} L_{2} C_{2}\right)+j \omega_{0} C_{2} r_{2}\right) \\
1
\end{array}\right]
\end{aligned}
$$

where

$$
\begin{aligned}
n_{11}\left(j \omega_{0}\right) & =\left(\left(-C_{2} L_{1} r_{2}-C_{2} L_{2} r_{1}\right) \omega_{0}^{2}+r_{1}\right) \\
& +j\left(-C_{2} L_{1} L_{2} \omega_{0}^{3}+\left(C_{2} r_{1} r_{2}+L_{1}\right) \omega_{0}\right), \\
n_{12}\left(j \omega_{0}\right) & =n_{21}\left(j \omega_{0}\right)=r_{1}+j \omega_{0} L_{1}, \\
n_{22}\left(j \omega_{0}\right) & =\left(\left(-C_{1} L_{1} r_{2}-C_{1} L_{2} r_{1}\right) \omega_{0}^{2}+r_{1}+r_{2}\right) \\
& +j\left(-C_{1} L_{1} L_{2} \omega_{0}^{3}+\left(C_{1} r_{1} r_{2}+L_{1}+L_{2}\right) \omega_{0}\right), \\
d\left(j \omega_{0}\right) & =\left(C_{1} C_{2} L_{1} L_{2} \omega_{0}^{4}+\left(-C_{1} C_{2} r_{1} r_{2}-C_{1} L_{1}-C_{2} L_{1}\right.\right. \\
& \left.\left.-C_{2} L_{2}\right) \omega_{0}^{2}+1\right)+j\left(\left(-C_{1} C_{2} L_{1} r_{2}-C_{1} C_{2} L_{2} r_{1}\right) \omega_{0}^{3}\right. \\
& \left.+\left(C_{1} r_{1}+C_{2} r_{1}+C_{2} r_{2}\right) \omega_{0}\right) .
\end{aligned}
$$

Using the circuit parameters in Table I results in

$$
\begin{gathered}
G=10^{-2} \cdot\left[\begin{array}{cc}
4.185+j 2.345 & 4.269+j 2.287 \\
4.269+j 2.287 & 10.469+j 5.219
\end{array}\right], \\
K=\left[\begin{array}{c}
24.526-j 0.953 \\
24.738-j 1.433
\end{array}\right] .
\end{gathered}
$$

Fig. 6 shows the feasibility and infeasibility regions on the $P_{1}-P_{2}$ plane for the condition $R_{P}(T)>0$. The graph was obtained taking fixed values for $P_{1}, P_{2}$ in the discretized 


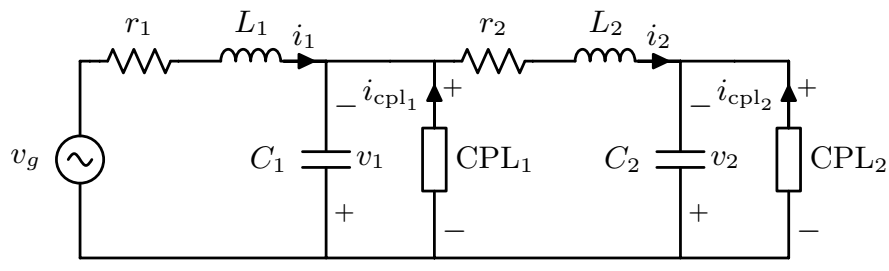

Fig. 5. LTI AC circuit with two CPLs.

set $[0,4000] \times[0,2000]$. We conclude that the blue area is contained in the set $\mathcal{P}_{\mathrm{FAQ}}^{\mathrm{I}}$. That is, for all values of $P_{1}$ and $P_{2}$ in this area the circuit of Fig. 5 does not admit a steady-state operating regime - this independently of the values of $Q_{1}$ and $Q_{2}$.

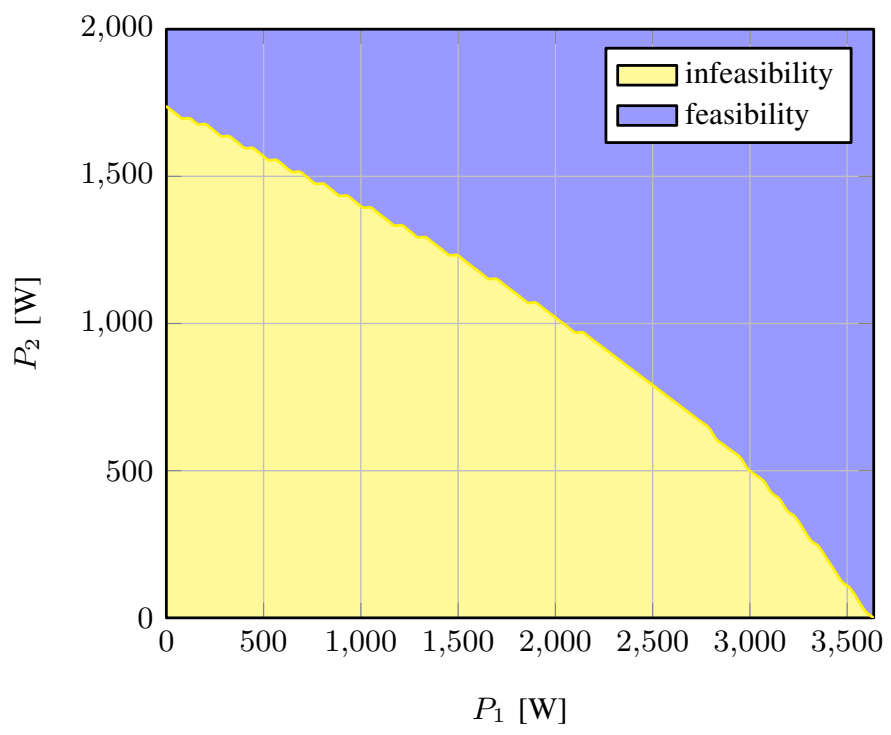

Fig. 6. Feasibility and infeasibility regions of $R_{P}(T)>0$ for the circuit of Fig. 5.

Fig. 7 shows the feasibility and infeasibility regions on the $Q_{1}-Q_{2}$ plane for the condition $R_{Q}(\bar{T})>0$. The gridding for $Q_{1}, Q_{2}$ was taken this time in the discretized set $[-5000,10000] \times[-5000,10000]$. The same conclusion as above applies to values of $Q_{1}$ and $Q_{2}$ in the blue area, which is contained in the set $\mathcal{Q}_{\mathrm{FAP}}^{\mathrm{I}}$.

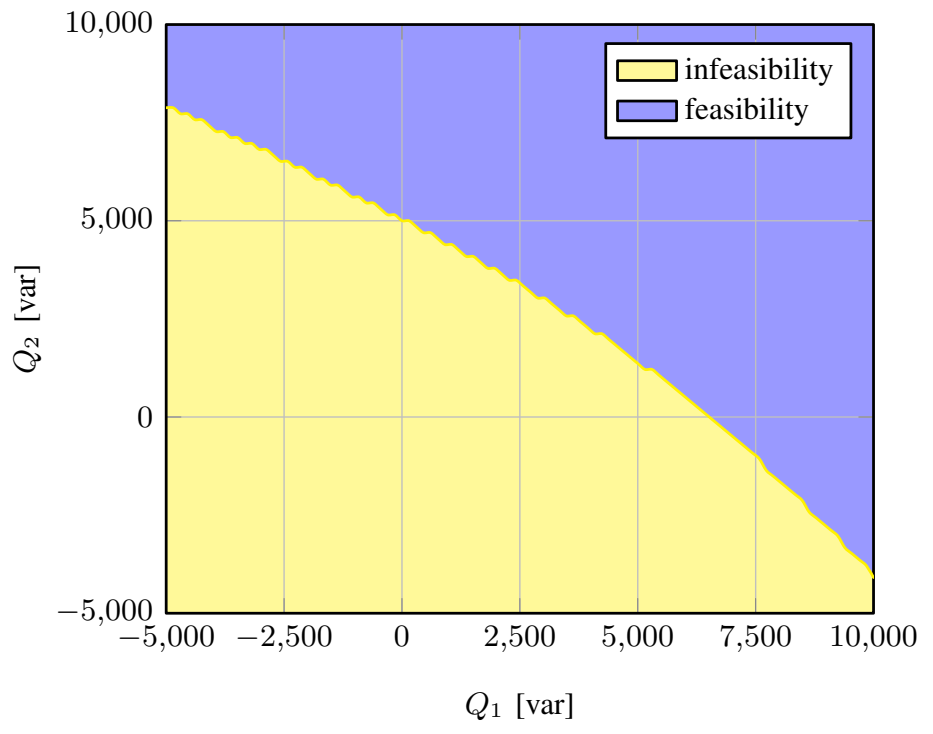

Fig. 7. Feasibility and infeasibility regions of $R_{Q}(\bar{T})>0$ for the circuit of Fig. 5.

Since we are dealing with a two port system non-feasibility of the LMI $R_{P}(T)>0$ in Proposition 2 is necessary and sufficient for existence of equilibria when $P$ is fixed and $Q$ is free-similarly $R_{Q}(\bar{T})>0$ is the necessary and sufficient test when $Q$ is fixed and $P$ is free. To corroborate this fact, we draw with a gray line in Fig. 8 the numerically exact boundary of existence of solutions for $\operatorname{Re}\left\{V_{i} I_{i}^{*}\right\}=-P_{i}$, for on the plane $P_{1}-P_{2}$. As predicted by the theory it exactly coincides (up to some numerical glitches) with the feasibility/infeasibility boundary for the condition $R_{P}(T)>0$, drawn also in gray but with a circle marker.

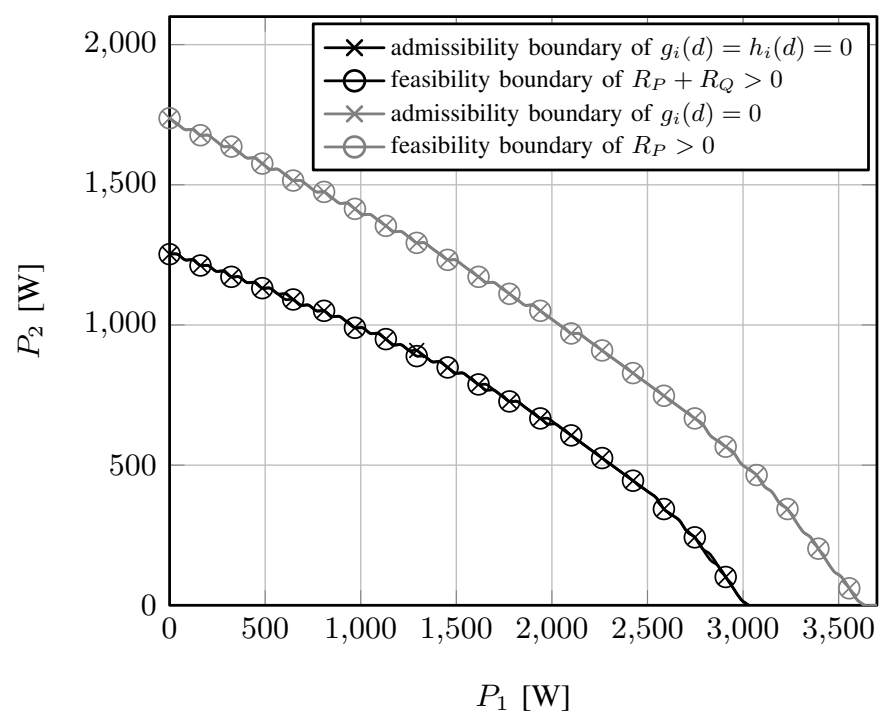

Fig. 8. Comparison between different boundaries of admissibility and feasibility for the two-port network of Fig. 5 on the $P_{1}-P_{2}$ plane.

Analogously, the numerically exact boundary of existence of solutions for $\operatorname{Im}\left\{V_{i} I_{i}^{*}\right\}=-Q_{i}$ on the plane $Q_{1}-Q_{2}$, is 
shown in Fig. 9 to coincide with the feasibility/infeasibility boundary for the condition $R_{Q}(\bar{T})>0$.

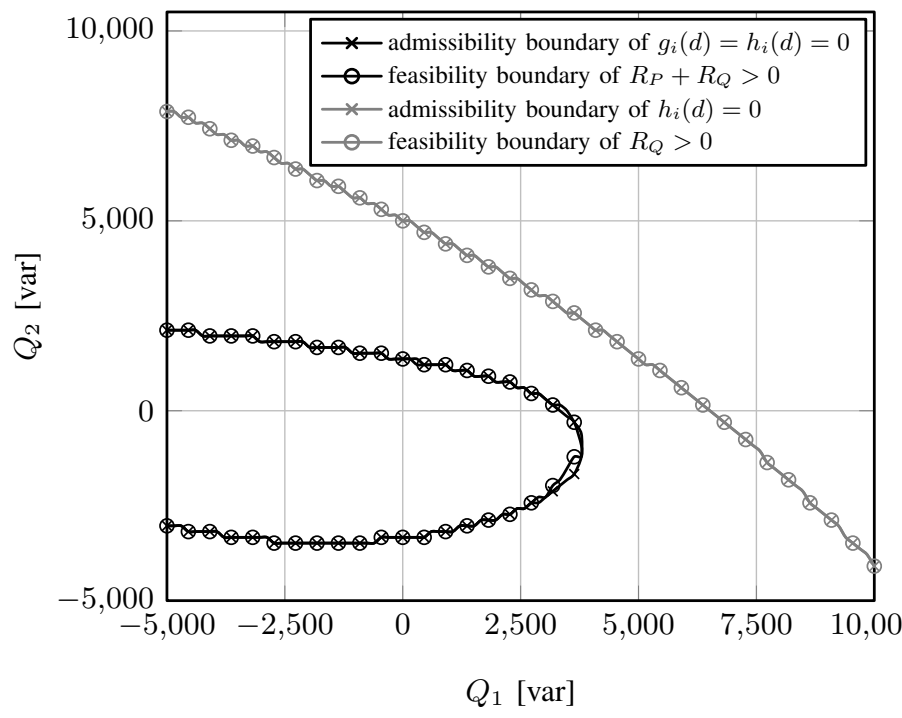

Fig. 9. Comparison between different boundaries of admissibility and feasibility for the two-port network of Fig. 5 on the $Q_{1}-Q_{2}$ plane.

It should be underscored that the admissibility region for $P$ and $Q$ is not the union of their separate admissible regions. That is, even if we take values for $P$ and $Q$ inside their respective admissible regions, i.e., under the gray curves in Figs. 8 and 9, respectively, this does not imply that the system will have an equilibrium for these CPLs. The reason is that such boundaries were obtained solving the real and the imaginary part of (4) independently and not simultaneously. The black curves with an ' $x$ ' marker in Figs. 8 and 9 represent the numerically exact boundaries of existence for (4), solving its real and imaginary parts simultaneously, and taking fixed values of $Q_{1}=1000$ and $Q_{2}=0$ for the former, and taking fixed values of $P_{1}=500$ and $P_{2}=500$ for the latter. It can be observed that in both cases the admissibility regions are reduced. However, they are always bounded by the feasibility/infeasibility boundaries of $R_{P}(T)>0$ and $R_{Q}(\bar{T})>0$.

Finally, the black curves with circle marker in Figs. 8 and 9 correspond to the feasibility/infeasibility boundaries of the LMI $R_{P}(T)+R_{Q}(\bar{T})>0$ plotted for some fixed values of $P$ and $Q$, respectively. Although not predicted by the theory, they coincide with the boundaries of numerically exact solution of the quadratic equations (4) for these fixed values-see Remark 6.

\section{CONCLUSions AND Future RESEARCH}

We have given in this paper necessary conditions for inadmissibility of CPLs, that is, values of their active and reactive power for which the network does not admit a sinusoidal steady state at a given frequency. For the case of one- or twoport networks and free active (or reactive) power components these conditions are also sufficient. Interestingly, for singleport networks the admissibility condition can be checked directly from the data of the problem, i.e., inequality (23). Similarly to the case of DC networks studied in [1], [7] the analysis boils down to the study of solvability of a set of quadratic equations. In the DC case these equations define mappings from $\mathbb{R}^{m}$ to $\mathbb{R}$, while in the $\mathrm{AC}$ case the mappings are from $\mathbb{C}^{m}$ to $\mathbb{C}$, giving rise to a different mathematical problem.

Current research is under way in three different directions. - Give conditions under which the LMI tests are necessary and sufficient as discussed in Remark 7. Also, similarly to the third point of Proposition 3, give conditions that can be checked directly from $G, K$ and $S$. These questions are related with the property of convexity of the image of the mappings defined by the quadratic equations and it has been thoroughly discussed in Section IV.A of [1]. Some encouraging results regarding this issue have been recently found and will be reported shortly.

- Assuming that a steady-state exists, to study its stability or attractivity properties. This task is carried out in [1] invoking Lyapunov's first method. In the present case, the analysis is much more complicated because, on one hand, the steadystate behavior is not a constant operating point but a periodic orbit. On the other hand, it is not clear how to characterise the behaviour of the CPLs in the time domain.

- Extend the analysis beyond the LTI network setting considered here. In particular, we are investigating the scenario of switched power converters with CPLs, a case studied in [1], [7] looking at the power balance equation.

\section{REFERENCES}

[1] N. Barabanov, R. Ortega, R. Griñó and B. Polyak, "On existence and stability of equilibria of linear time-invariant systems with constant power loads," IEEE Transactions on Circuits and Systems I: Regular Papers, Vol. 63, No. 1, pp. 114-121, 2016.

[2] C. A. Desoer and E. Kuh, Basic Circuit Theory, McGraw-Hill Book Company, 1969.

[3] A. Emadi, A. Khaligh, C. H. Rivetta and G. A. Williamson, "Constant power loads and negative impedance instability in automotive systems: definition, modeling, stability, and control of power electronic converters and motor drives," in IEEE Transactions on Vehicular Technology, vol. 55, no. 4, pp. 1112-1125, 2006.

[4] A. Khaligh, A. M. Rahimi and A. Emadi, "Modified Pulse-Adjustment Technique to Control DC/DC Converters Driving Variable ConstantPower Loads," in IEEE Transactions on Industrial Electronics, vol. 55, no. 3, pp. 1133-1146, 2008.

[5] D. Marx, P. Magne, B. Nahid-Mobarakeh, S. Pierfederici and B. Davat, "Large signal stability analysis tools in dc power systems with constant power loads and variable power loads-A review," IEEE Trans. Power Electron., Vol. 27, No. 4, pp. 1773-1787, 2012.

[6] B. Polyak, "Convexity of quadratic transformations and its use in control and optimization," Journal of Optimization Theory and Applications, Vol. 99, No. 3, pp. 553-583, 1998.

[7] S. Sanchez, R. Ortega, R. Griñó, G. Bergna and M. Molinas-Cabrera, "Conditions for existence of equilibrium points of systems with constant power loads," IEEE Transactions on Circuits and Systems I: Regular Papers, Vol. 61, No. 7, pp. 2204-2211, 2014.

[8] S. Singh, A. R. Gautam, and D. Fulwani, "Constant power loads and their effects in DC distributed power systems: A review," Renewable and Sustainable Energy Reviews, Vol. 72, pp. 407-421, 2017.

[9] F. Uhlig, "A recurring theorem about pairs of quadratic forms and extensions: A survey," Linear Algebra and its Applications, Vol. 25, pp. 219-237, 1979. 


\section{APPENDIX}

\section{A. Proof of LEMMA 2}

To streamline the presentation we define the set

$$
\mathcal{E}_{v}:=\left\{x \in \mathbb{R}^{n} \mid v_{k}(x)=0\right\},
$$

where $v_{k}(x)$ is defined in (11).

The proof of the implication (13) is established by contraposition. Therefore, suppose that $\mathcal{E}_{v}$ is not empty. We will then prove that there are no real numbers $t_{k}$ such that

$$
\sum_{k=1}^{\ell} t_{k} \mathcal{A}_{k}>0
$$

Let us take $x \in \mathcal{E}_{v}$ and $t_{k}$ arbitrary. Since $v_{k}(x)=0$, then $t_{k} v_{k}(x)=0$. This implies that

$$
\sum_{k=1}^{\ell} t_{k} v_{k}(x)=0
$$

Define the vector $z:=\operatorname{col}(x, 1) \in \mathbb{R}^{n+1}$. Then, using (12), equation (27) can be represented in matrix form as

$$
z^{\top}\left(\sum_{k=1}^{\ell} t_{k} \mathcal{A}_{k}\right) z=0 .
$$

Since $z$ is a non-zero vector, (28) contradicts inequality (26) for an arbitrary selection of $t_{k}$, completing the proof.

\section{B. PROOF OF LEMMA 3}

The proof invokes Finsler's Lemma, in particular Statement I of the Main Theorem in [9] that, using the notation of Lemma 3 , reads as follows:

$$
\begin{array}{r}
\exists t_{1}, t_{2} \in \mathbb{R} \mid t_{1} \mathcal{A}_{1}+t_{2} \mathcal{A}_{2}>0 \\
\Leftrightarrow\left\{\eta \in \mathbb{R}^{n+1} \mid \eta^{\top} \mathcal{A}_{1} \eta=\eta^{\top} \mathcal{A}_{2} \eta=0\right\}=\{\mathbf{0}\},
\end{array}
$$

where $\mathcal{A}_{1}, \mathcal{A}_{2}$ are given by (12). Notice that this theorem pertains to homogeneous quadratic forms while, because of the presence of the terms $2 x^{\top} b_{k}+c_{k}$, the equations of interest to us, e.g., (11), are non-homogeneous.

To adapt Finsler's Theorem to handle this case we define two real mappings $w_{1}, w_{2}: \mathbb{R}^{n} \times \mathbb{R} \rightarrow \mathbb{R}$

$$
\begin{aligned}
& w_{1}(x, y):=x^{\top} A_{1} x+2 x^{\top} b_{1} y+c_{1} y^{2} \\
& w_{2}(x, y):=x^{\top} A_{2} x+2 x^{\top} b_{2} y+c_{2} y^{2}
\end{aligned}
$$

and the set

$$
\mathcal{E}_{w}:=\left\{(x, y) \in \mathbb{R}^{n} \times \mathbb{R} \mid w_{1}(x, y)=w_{2}(x, y)=0\right\} .
$$

First, we will prove that under condition (14), the following equivalence holds:

$\mathcal{E}_{v}=\left\{x \in \mathbb{R}^{n} \mid v_{1}(x)=v_{2}(x)=0\right\}=\varnothing \Leftrightarrow \mathcal{E}_{w}=\{(\mathbf{0}, 0)\}$.

$(\Rightarrow)$ The proof is by contraposition, therefore, we suppose there is a non-zero vector $(\bar{x}, \bar{y}) \in \mathcal{E}_{w}$. Consider first the case when $\bar{y}=0$, then $\bar{x} \neq 0$ necessarily. Since $w_{1}(\bar{x}, 0)=$ $w_{2}(\bar{x}, 0)=0$ This implies that

$$
\bar{x}^{\top} A_{1} \bar{x}=\bar{x}^{\top} A_{2} \bar{x}=0 .
$$

Let $s_{1}, s_{2} \in \mathbb{R}$ arbitrary. Then, the following expression holds

$$
\bar{x}^{\top}\left(s_{1} A_{1}+s_{2} A_{2}\right) \bar{x}=0 .
$$

However, this contradicts (14). Assume now that $\bar{y} \neq 0$. In this case it follows that $\frac{\bar{x}}{\bar{y}} \in \mathcal{E}_{v}$. Hence, $\mathcal{E}_{v} \neq \varnothing$, completing the proof.

$(\Leftarrow)$ Once again by contraposition. Hence, suppose that $\mathcal{E}_{v} \neq \varnothing$ and take $\bar{x} \in \mathcal{E}_{v}$, then $(\bar{x}, 1)$ is a non-zero vector in $\mathcal{E}_{w}$ and, consequently, $\mathcal{E}_{w} \neq\{\mathbf{0}\}$, completing the proof.

We are in position now to prove the implication (15). Suppose $\mathcal{E}_{v}=\varnothing$. Then, the equivalence (31) implies that $\mathcal{E}_{w}=\{(\mathbf{0}, 0)\}$. Recalling that

$$
w_{1}(x, y)=\eta^{\top} \mathcal{A}_{1} \eta, w_{2}(x, y)=\eta^{\top} \mathcal{A}_{2} \eta
$$

with $\eta:=(x, y)$, Finsler's Lemma ensures the existence of $t_{1}, t_{2} \in \mathbb{R}$ such that

$$
t_{1} \mathcal{A}_{1}+t_{2} \mathcal{A}_{2}>0 \text {. }
$$

Hence, the lemma is proved.

\section{Proof of Proposition 1}

Define the following $(2 m+1) \times(2 m+1)$ real matrices

$$
\hat{\mathcal{A}}_{i}:=\left[\begin{array}{cc}
\hat{A}_{i} & \hat{b}_{i} \\
\hat{b}_{i}^{\top} & 2 P_{i}
\end{array}\right], \hat{\mathcal{B}}_{i}:=\left[\begin{array}{cc}
\hat{B}_{i} & \hat{q}_{i} \\
\hat{q}_{i}^{\top} & 2 Q_{i}
\end{array}\right] .
$$

Notice that

$$
\begin{aligned}
& g_{i}(d)=\left[\begin{array}{ll}
d^{\top} & 1
\end{array}\right] \hat{\mathcal{A}}_{i}\left[\begin{array}{l}
d \\
1
\end{array}\right] \\
& h_{i}(d)=\left[\begin{array}{ll}
d^{\top} & 1
\end{array}\right] \hat{\mathcal{B}}_{i}\left[\begin{array}{l}
d \\
1
\end{array}\right] .
\end{aligned}
$$

Suppose there exist $t_{i}, \bar{t}_{i}$ such that ${ }^{4}$

$$
\sum_{i=1}^{m} t_{i} \hat{\mathcal{A}}_{i}+\sum_{i=1}^{m} \bar{t}_{i} \hat{\mathcal{B}}_{i}>0 .
$$

Referring to Lemma 2 with $\ell=2 m, x=d$ and

$$
v_{1}=g_{1}, \ldots, v_{m}=g_{m}, v_{m+1}=h_{1}, \ldots, v_{2 m}=h_{m},
$$

we conclude that there are no solutions for the system

$$
g_{i}(d)=h_{i}(d)=0 .
$$

This implies that there is no sinusoidal steady-state for the network. The proof is completed noting that

$$
\sum_{i=1}^{m} t_{i} \hat{\mathcal{A}}_{i}=R_{P}(T), \sum_{i=1}^{m} \bar{t}_{i} \hat{\mathcal{B}}_{i}=R_{Q}(\bar{T}) .
$$

\section{Proof of Proposition 2}

Consider the matrix $R_{P}$, given in (17), for $T=I_{m}$. Notice that the uppermost corner of $R_{P}$ is given by $M$, defined in (19). Since $M>0$ we have that $R_{P}>0$ if and only if the Schur complement of $M$ is positive. The proof is completed noting that the latter is equivalent to (20).

\footnotetext{
${ }^{4} \mathrm{We}$ introduced the symbol $\bar{t}_{i}$ for ease of reference in the subsequent material.
} 


\section{E. Proof of Proposition 3}

The equivalence between the first two statements is a direct corollary of the last statement in Lemma 3, which states that for $\ell=2$ (that is $m=1$ )

$$
g_{1}(d)=h_{1}(d)=0 \Leftrightarrow \exists t_{1}, \bar{t}_{1} \mid t_{1} \mathcal{A}_{1}+\bar{t}_{1} \mathcal{B}_{1}>0 .
$$

The proof is completed invoking the identity (36) with $T=$ $t_{1}, \bar{T}=\bar{t}_{1}$.

We now proceed to show the equivalence with the last statement. Towards this end, let us re-write model of the network in complex form as

$$
G|I|^{2}+I^{*} K+S=0 .
$$

Inequality (2) implies that $G \neq 0$. Divide (37) by $G$ and split it into real and imaginary parts. Then, the complex equation (37) is equivalent to

$$
\begin{array}{r}
x^{2}+y^{2}+a x+b y+P^{\prime}=0 \\
b x-a y+Q^{\prime}=0
\end{array}
$$

where

$$
\frac{K}{G}:=a+j b, \quad \frac{S}{G}:=P^{\prime}+j Q^{\prime}, \quad I:=x+j y .
$$

Let us suppose that $a \neq 0$, then from (39) we can get $y$ as

$$
y=\frac{b x+Q^{\prime}}{a} \text {. }
$$

Substituting the above value for $y$ into (38) we get the following quadratic equation for $x$

$$
\left(a^{2}+b^{2}\right) x^{2}+\left[2 b Q^{\prime}+a\left(a^{2}+b^{2}\right)\right] x+\left(Q^{\prime 2}+a b Q^{\prime}+a^{2} P^{\prime}\right)=0 .
$$

This equation has a real solution if and only if its discriminant is non-negative, that is, if and only if

$$
\left(2 b Q^{\prime}+a\left(a^{2}+b^{2}\right)\right)^{2} \geq 4\left(a^{2}+b^{2}\right)\left(Q^{\prime 2}+a b Q^{\prime}+a^{2} P^{\prime}\right) .
$$

If we develop the left and right-hand side expressions, then the inequality above is equivalent to

$$
\begin{gathered}
4 b^{2} Q^{\prime 2}+4 a b Q^{\prime}\left(a^{2}+b^{2}\right)+a^{2}\left(a^{2}+b^{2}\right)^{2} \\
\geq \\
4 a^{2} Q^{\prime 2}+4 b^{2} Q^{\prime 2}+4 a b Q^{\prime}\left(a^{2}+b^{2}\right)+4 a^{2} P^{\prime}\left(a^{2}+b^{2}\right)
\end{gathered}
$$

which can be written in a simplified form as

$$
\left(a^{2}+b^{2}\right)^{2}-4 P^{\prime}\left(a^{2}+b^{2}\right)-4 Q^{\prime 2} \geq 0 .
$$

This expression holds if and only if

$$
\left(a^{2}+b^{2}\right) \geq 2\left(P^{\prime}+\sqrt{P^{\prime 2}+Q^{\prime 2}}\right) .
$$

Let us note that

$$
\begin{aligned}
a^{2}+b^{2} & =\frac{|K|^{2}}{|G|^{2}}, \\
P^{\prime} & =\frac{P \operatorname{Re}\{G\}+Q \operatorname{Im}\{G\}}{|G|^{2}}, \\
Q^{\prime} & =\frac{Q \operatorname{Re}\{G\}-P \operatorname{Im}\{G\}}{|G|^{2}} .
\end{aligned}
$$

Then, inequality (42) can be equivalently written in the original coefficients as in in (23).
Now let us suppose that $a=0$. If $b \neq 0$, then from (39) we have that

$$
x=-\frac{Q^{\prime}}{b} .
$$

If we substitute this value of $x$ into (38), we get a quadratic equation for $y$ given by

$$
b^{2} y^{2}+b^{3} y+\left(Q^{\prime 2}+b^{2} P^{\prime}\right)=0 .
$$

This equation has a real solution if and only if its discriminant is non negative, that is if and only if

$$
b^{6}-4 b^{2}\left(Q^{\prime 2}+b^{2} P^{\prime}\right) \geq 0 .
$$

This inequality is equivalent to

$$
\left(b^{2}\right)^{2}-4 P^{\prime}\left(b^{2}\right)-4 Q^{\prime 2} \geq 0,
$$

which holds if and only if

$$
b^{2} \geq 2\left(P^{\prime}+\sqrt{P^{\prime 2}+Q^{\prime 2}}\right) .
$$

Clearly, the above expression corresponds with (42) by taking $a=0$.

To conclude the proof, let us suppose that $a=0$ and $b=0$ simultaneously. In this case, system conformed by equations (38) and (39), has a solution if and only if $Q^{\prime}=0$ and $P^{\prime} \leq 0$. This last case has little practical relevance since if $a=0$ and $b=0$ simultaneously, then $K=0$, which would correspond to a single-port network without a source.

\section{Proofs of Propositions 4 AND 5}

The proofs follow as direct corollaries of Lemma 3. First, notice that

$$
\begin{aligned}
& g_{1}(d)=g_{2}(d)=0 \Leftrightarrow \exists t_{1}, t_{2} \text { such that } t_{1} \mathcal{A}_{1}+t_{2} \mathcal{A}_{2}>0 \\
& h_{1}(d)=h_{2}(d)=0 \Leftrightarrow \exists \bar{t}_{1}, \bar{t}_{2} \text { such that } \bar{t}_{1} \mathcal{B}_{1}+\bar{t}_{2} \mathcal{B}_{2}>0,
\end{aligned}
$$

and

$$
\begin{aligned}
t_{1} \mathcal{A}_{1}+t_{2} \mathcal{A}_{2} & =R_{P}\left(\operatorname{diag}\left\{t_{1} \cdot t_{2}\right\}\right) \\
\bar{t}_{1} \mathcal{B}_{1}+\bar{t}_{2} \mathcal{B}_{2} & =R_{Q}\left(\operatorname{diag}\left\{\bar{t}_{1} \cdot \bar{t}_{2}\right\}\right) .
\end{aligned}
$$

It only remains to verify (14). For Proposition 4 we have

$$
\hat{A}_{1}+\hat{A}_{2}=M,
$$

with $M$, given in (19), being positive definite. Therefore, (14) is satisfied with $s_{1}=s_{2}=1$. On the other hand, for Proposition 5 it is not clear when there exists $\hat{s}_{1}, \hat{s}_{2} \in \mathbb{R}$ such that

$$
\hat{s}_{1} \hat{B}_{1}+\hat{s}_{2} \hat{B}_{2}>0,
$$

with $\hat{B}_{1}, \hat{B}_{2}$ given in (9). Therefore, it is necessary to impose assumption (24). 


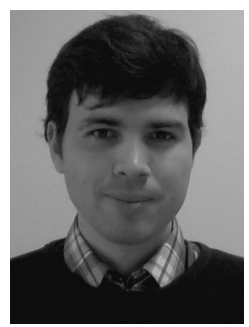

Juan E. Machado received the B.Sc. degree in electromechanical engineering in 2012 from Instituto Tecnológico de La Paz, La Paz, México and the M.Sc. degree in applied mathematics in 2015 from Centro de Investigación en Matemáticas, Guanajuato, México. Currently, he is a Ph.D student at Université Paris Sud - Centrale Supélec, Gif-SurYvette, France. His interests include modeling and control of electromechanical systems.

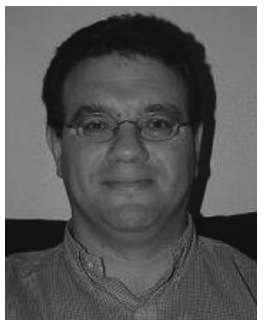

Robert Griñó (M'99, SM'12) received the M.Sc. degree in electrical engineering and the Ph.D. degree in automatic control from the Universitat Politècnica de Catalunya (UPC), Barcelona, Spain, in 1989 and 1997, respectively. From 1990 to 1991, he was a Research Assistant with the Instituto de Cibernética, UPC. From 1992 to 1998, he was an Assistant Professor with the Automatic Control Department, Universitat Politècnica de Catalunya, where he has been an Associate Professor since 1998. His research interests include digital control, nonlinear control, stability theory and control of power electronics converters. Dr. Griñó is an affiliate member of International Federation of Automatic Control (IFAC) and a member of the Spanish Society on Automation and Control-IFAC.

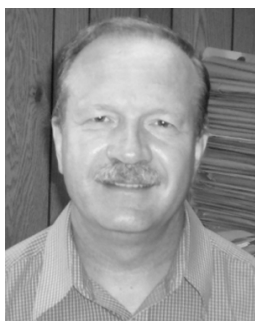

Nikita Barabanov received the M.Sc. and Ph.D. degrees in mathematics from Leningrad University, USSR, in 1976 and 1979 respectively, and the Doctor of Science Degree from Kiev Institute of $\mathrm{Cy}$ bernetics, USSR, in 1990. Between 1979 and 2002 he has been with St. Petersburg Electrotechnical University, Russia, where he has got a position of Full Professor since 1991. Since 2002 he is with the department of Mathematics of North Dakota State University. He had Visiting Professor's positions in Ford Motor Company (USA), Newcastle University (Australia), Perth University (Australia), and Supelec (Paris). He is the author of more than 70 scientific papers in peer-reviewed journals. He was a supervisor of Ph.D. students in applied mathematics and control theory, and member of Ph.D. committees in Russia, Australia, France, and USA. He was a winner of many grants in control theory and differential equations. In 1980 he has got the Award of the Leningrad Mathematical Society. He is a member of Leningrad Mathematical Society and American Mathematical Society.

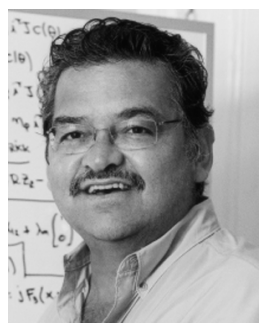

Romeo Ortega (S'81, M'85, SM'98, F'99) was born in Mexico. He obtained his BSc in Electrical and Mechanical Engineering from the National University of Mexico, Master of Engineering from Polytechnical Institute of Leningrad, USSR, and the Docteur D`Etat from the Politechnical Institute of Grenoble, France in 1974, 1978 and 1984 respectively.

He then joined the National University of Mexico, where he worked until 1989. He was a Visiting Professor at the University of Illinois in 1987-88 and at McGill University in 1991-1992, and a Fellow of the Japan Society for Promotion of Science in 1990-1991. He has been a member of the French National Research Council (CNRS) since June 1992. Currently he is a Directeur de Recherche in the Laboratoire de Signaux et Systemes (CentraleSupelec) in Gif-sur-Yvette, France. His research interests are in the fields of nonlinear and adaptive control, with special emphasis on applications.

Dr Ortega has published three books and more than 290 scientific papers in international journals, with an h-index of 73 . He has supervised more than $30 \mathrm{PhD}$ thesis. He is a Fellow Member of the IEEE since 1999 and an IFAC Fellow since 2016. He has served as chairman in several IFAC and IEEE committees and participated in various editorial boards of international journals.

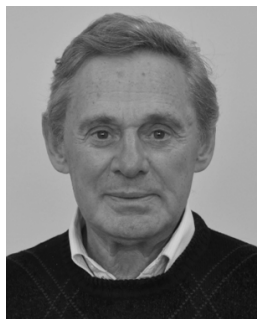

Boris Polyak obtained his $\mathrm{PhD}$ in mathematics from Moscow State University, USSR in 1963 and the Doctor of Science Degree from Moscow Institute for Control Science in 1986. Between 1963 and 1971 he has been with Moscow State University, and since 1971 he is with Moscow Institute for Control Science. He has been Head of Tsypkin laboratory and currently he is Chief Researcher at the Institute. Simultaneously he is a Professor at Moscow Institute for Physics and Technology. He had visiting positions at Universities of USA, France, Italy, Israel, Finland and Taiwan. His research interests are in the fields of optimization and control with emphasis on stochastic optimization and robust control. Prof. Polyak has published four books and more than 200 scientific papers in international journals. He has supervised $25 \mathrm{PhD}$ thesis, he is a member of numerous international and Russian editorial boards. Chair of Organizing Committees of 1 - 7-th Traditional Schools for Young Researchers "Control, Information and Optimization". He is IFAC Fellow, recipient of Gold Medal EURO-2012 of European Operational Research Society. 